\title{
A Review on Radiation Damage in Concrete for Nuclear Facilities: From Experiments to Modeling
}

\author{
Beatrice Pomaro \\ Department of Civil, Environmental and Architectural Engineering, University of Padova, via F. Marzolo 9, 35131 Padova, Italy \\ Correspondence should be addressed to Beatrice Pomaro; beatrice.pomaro@dicea.unipd.it
}

Received 24 December 2015; Accepted 19 July 2016

Academic Editor: Anna Pandolfi

Copyright (C) 2016 Beatrice Pomaro. This is an open access article distributed under the Creative Commons Attribution License, which permits unrestricted use, distribution, and reproduction in any medium, provided the original work is properly cited.

\begin{abstract}
Concrete is a relatively cheap material and easy to be cast into variously shaped structures. Its good shielding properties against neutrons and gamma-rays, due to its intrinsic water content and relatively high-density, respectively, make it the most widely used material for radiation shielding also. Concrete is so chosen as biological barrier in nuclear reactors and other nuclear facilities where neutron sources are hosted. Theoretical formulas are available in nuclear engineering manuals for the optimum thickness of shielding for radioprotection purposes; however they are restricted to one-dimensional problems; besides the basic empirical constants do not consider radiation damage effects, while its long-term performance is crucial for the safe operation of such facilities. To understand the behaviour of concrete properties, it is necessary to examine concrete strength and stiffness, water behavior, volume change of cement paste, and aggregate under irradiated conditions. Radiation damage process is not well understood yet and there is not a unified approach to the practical and predictive assessment of irradiated concrete, which combines both physics and structural mechanics issues. This paper provides a collection of the most distinguished contributions on this topic in the past 50 years. At present a remarkable renewed interest in the subject is shown.
\end{abstract}

\section{Introduction}

Despite the increasing interest in renewable energy, nuclear power still has an important role in the energy supply worldwide. Almost $12 \%$ of world's electricity is generated by nuclear power plants; nuclear energy is used by 30 countries in the world, for total 439 units, 64 new ones being under design. Europe depends on nuclear power for more than one-quarter of its electricity; in fact almost $30 \%$ of electricity demand is satisfied this way; $40 \%$ comes from fossil fuel and the remaining from renewables. 131 nuclear power reactors were operating in 2014 in Europe and nuclear plant construction is currently underway in three EU member states: Finland, France, and Slovakia [1].

Understanding the conditions that lead to concrete deterioration is object of a new attention worldwide in matter of nuclear plants. In USA many plants have already had a life extension from 40 to 60 years and serious consideration is now being given to a further extension to 80 years. In fact according to the Atomic Energy Act of 1954 and the Nuclear Regulatory Commission regulations the operating licenses for commercial power reactors are issued for 40 years and can be renewed for additional 20 years with no limit to the number of renewals.

Japan has now entered the period of safety management of nuclear plants; 20 nuclear power plants in Japan have undergone an examination called Plant Life Management by the Japanese government to determine their ability to carry on continuous operation. So in this country work is in progress to improve a quantitative evaluation method based on the time-dependent performance of structures and their components in relation to the deterioration mechanism, which could serve as a maintenance program to extend the operational period of up to 20 years in the near future.

Most of the EU's operational nuclear plants were built in the 1970s and 1980s and were designed to last for around 40 years; therefore they are due to retire by the 2020s unless they get extensions [2]. Some of the oldest reactors are in Belgium, two of which just reached age 40 , and 10 extra years has been recently approved for them until 2025 [3]; France is the EU's nuclear leader, its 58 reactors producing nearly three-quarters of the country's electricity; this country also is considering 
whether to extend their lifespan to 50 years in 2018 or 2019 [4].

Nuclear reactors, however, are not the only structural type undergoing nuclear radiation damage which must address durability considerations.

For instance, radioisotopes are extensively used in medicine; in over 10,000 hospitals worldwide radiotherapy is the way in which some medical conditions are treated, using radiation to weaken or destroy particular targeted cells; these facilities as well require the design of bunker-type rooms, able to sustain photon and neutron radiation.

This paper is aimed at picturing out state of the art of the most relevant contributions from the late 1950s up to now on the topic of radiation damage of concrete employed for nuclear shielding, from the phenomenological evidences to the modeling aspects.

Many contemporary authors agree that the technical information necessary for determining an appropriate threshold value of nuclear radiation in assessing the soundness of concrete structures under irradiation conditions is poor, especially in regard to concrete durability evaluation. Consequently, further experimental tests are needed. On the other hand, from the first studies some advancements on the interaction between concrete and neutrons or gamma-rays have been made, which would now need implementation in predictive models of concrete deterioration and validation against representative laboratory tests to evaluate the related rate effects.

The understanding of radiation effects on concrete was largely based on Hilsdorf and coauthors' curves compiled in 1978 [5] up to a few years ago, when the need to develop comprehensive safety evaluation systems for aging nuclear power plants has become a compelling issue.

Fujiwara et al. [6] have recently conducted an irradiation test on concrete specimens simulating the exercise scenario of a boiling water reactor in Japan (maximum temperature $65^{\circ} \mathrm{C}$; maximum neutron fluence $12 \times 10^{18} \mathrm{n} / \mathrm{cm}^{2}(E>0.1 \mathrm{MeV})$ ) by far consistently with a 60 -year operation. With this work they confirmed that, within the range of radiation doses adopted in the study, radiation exposure did not significantly affect the basic long-term material characteristics; on the other hand the tendency pictured in [5] in terms of the decrease of concrete compressive strength in response to rise in neutron fluence was not detected, due to some critical observations supported also by [7-9].

In fact, despite the big effort in compiling a first data base of experimental results on irradiated concrete, the applicability of the reference values by Hilsdorf and coauthors to the soundness evaluation of concrete in light-water reactors is questioned in [8], basically in reason of some inconsistency of the experimental conditions with the real irradiation conditions (namely, Portland cement for the concrete mix; irradiation from fast neutron spectrum $(E>0.1 \mathrm{MeV})$; temperature field less than $65^{\circ} \mathrm{C}$ ); in particular the experimental database in [5] is said to show wide scattering due to different choices on measured properties (bending strength instead of compressive strength or bending tension strength instead of splitting tension strength), binder type of concrete, specimen size (even dimensions of $8-15 \mathrm{~mm}$ ), temperature of concrete $\left(100^{\circ} \mathrm{C}\right.$ or higher up to $550^{\circ} \mathrm{C}$ ), and neutron spectrum (slow or fast neutrons or not available spectrum).

The work by Field et al. [10] very recently expanded the data collection provided in [5] with those coming from later experimental campaigns on neutron-irradiated concrete and mortars; it provides a complete up to date database to start from to investigate the key variables associated with radiation-induced degradation in concrete structures. Effects on concrete in terms of compressive and tensile strength, modulus of elasticity, weight loss, and volumetric change are here plotted and classified under the applied laboratory conditions. It is here pointed out for a wider data collection that the effects of some important parameters such as the neutron fluence magnitude at which concrete deterioration becomes significant, the neutron fluence energy cutoff associated with the threshold fluence level, and the effect of high concrete temperature during irradiation, to mention a few, cannot be readily separated out and clearly evaluated from the existing data.

\section{Phenomenological Aspects on Irradiated Concrete}

Radiation on shielding materials is known to affect them deep in their chemical structure. In metals radiation leads to the displacements of atoms from their equilibrium lattice sites, causing lattice defects, which are responsible for an increase of hardness but also embrittlement, and so loss of ductility [11-13]. In polymers the formation of additional cross-links is due to the surplus energy brought by radiation [14]. In geomaterials, like concrete, nuclear radiation leads to the break of atomic bonds, which is supposed to explain the decay in the mechanical properties envisaged in exposed concrete $[15,16]$ as it will be illustrated in the following.

The first comprehensive collection of published experimental data on the effects of nuclear radiation on the properties of concrete dates back to Hilsdorf et al. [5] and Kaplan [17], with the recent observations on the first work already mentioned in the previous section. It stands out that up to integrated neutron fluence of the order of $10^{19} \mathrm{n} / \mathrm{cm}^{2}$ the effects of the irradiation are relatively small, while higher fluence may have detrimental effects on concrete compressive and tensile strength and modulus of elasticity.

In particular, distinguishing between fast or slow neutrons, Gray [18] found that for fast neutron fluence between $7 \times 10^{18}$ and $3 \times 10^{19} \mathrm{n} / \mathrm{cm}^{2}$ the modulus of irradiated concrete was between $10 \%$ and $20 \%$ less than that of nonirradiated unheated concrete. Alexander [19] reported similar reductions in values of elastic modulus for slow neutron fluence of about $2 \times 10^{19} \mathrm{n} / \mathrm{cm}^{2}$. Negligible changes in mechanical properties and weight/volume were found for fast neutrons with $E>1 \mathrm{MeV}$ at a fluence of $3 \times 10^{18} \mathrm{n} / \mathrm{cm}^{2}$ by Stoces et al. [20].

There is also evidence that concrete hardens under irradiation $[21,22]$ : from these studies the interference of penetrating ionizing radiation with the process of setting of cement paste has been found in the form of increased compressive 
and tensile strength, especially at the very beginning of the hardening period.

Almost in all the cases in which samples were tested to investigate the effects of neutron radiation, the concrete samples were also exposed to secondary gamma radiation, produced by nuclear reactions. Not many tests are in literature on the effects of gamma radiation alone without the simultaneous exposure to neutron radiation. Alexander [19] reports that for gamma radiation doses of about $10^{10} \mathrm{rad}$ there is no reduction in the compressive strength of concrete, when compared with the strength of companion specimens which have been neither irradiated nor heated; there is, however, evidence of reductions between $25 \%$ and $60 \%$ in compressive strength for doses exceeding $10^{11} \mathrm{rad}$ [23]. In this last test campaign the specimens were immersed in demineralized water in order to shield them against neutrons and study the effect of gamma radiation alone, but demineralized water was found to contribute to deteriorating concrete and after several years of exposure the surface of the irradiated samples was partially destroyed.

Soo and Milian [24] found that a loss in the compressive strength could occur at gamma doses that are much less than the threshold dose of $2 \times 10^{8} \mathrm{~Gy}\left(2 \times 10^{10} \mathrm{rad}\right)$. They postulated that the loss of strength could be connected with the radiolysis of the water of hydration in the cement as well as pore water. A loss of hydrogen and oxygen radiolytic species during irradiation would decrease the level of cement hydration and thus the strength of the cement.

The experimental campaign in [25] on concrete under gamma irradiation allowed concluding that the interaction with the shielding material leads to lowering both its strength and its porosity. The mechanism is explained to happen as a series of chemical reactions within the material, starting from the radiolysis of water and ending with the formation of calcite $\left(\mathrm{CaCO}_{3}\right)$; crystallites of calcite grow into pores, decreasing their size and destroying the tobermorite gel, a calcium silicate hydrate mineral responsible for concrete strength, by their crystallization pressure.

Another experimental study was conducted by Vodák et al. [26] to investigate further on the porosity variation in cement paste under gamma irradiation and under natural carbonation.

The radiolytic process seems to be confirmed by more recent studies [27], taking advantage of X-ray diffraction and scanning electron microscopy methods. The absorption of gamma radiation is found to induce the amorphization of cement hydrates and finally cause their decomposition. Moreover, bubbles were envisaged after irradiation exposure, which must be the effect of separation of the chemically bonded water, and numerous cracks in the cement matrix were also observed.

The presence of calcium is pointed out as a major cause for the progression of radiolysis; in fact the withdrawal of calcium under gamma-rays is responsible for the formation of $\mathrm{CaO}_{2} \cdot 8 \mathrm{H}_{2} \mathrm{O}$, a highly insoluble phase which forms at the expense of portlandite and ettringite, leading to their elimination in case of very high radiation doses [28].

The basic mechanisms controlling the radiolysis in cementitious matrices are reviewed in the specific context of gamma irradiation in [29]. Two main outcomes result from water radiolysis: (i) buildup of internal gas overpressure (mainly hydrogen and oxygen) that may lead to cracking and/or explosive gas mixture when hydrogen gas is mixed with atmospheric oxygen in fraction of $4 \%$ and (ii) corrosion of steel bars in the long-term due to the attack by oxidizing products at the steel-concrete interface [30].

Thermal expansion coefficient, thermal conductivity, and shielding properties appear to be little affected by radiation. As for the coefficient of thermal expansion, Hilsdorf et al. [5] and Granata and Montagnini [21] indicate that for neutron fluence less than $5 \times 10^{19} \mathrm{n} / \mathrm{cm}^{2}$ there is no significant difference between the coefficient of thermal expansion of neutron-irradiated concrete samples and the coefficient of nonirradiated samples subjected to high temperature. Also, radiation energy is converted to heat when absorbed by a shield. The heat generated by irradiation can reach quite high temperatures, depending on the shielding material used and the configuration of the shielding structure. Hilsdorf et al. [5] found that temperatures even of the order of $250^{\circ} \mathrm{C}$ developed in some of the investigations he summarized, which is high enough to generate considerable damage in shielding materials.

An extensive study on the effect of elevated temperature exposure on properties and shielding effectiveness is reported in [31].

As for creep experiments under irradiation, limited reference data exist: $[18,32]$ for neutron and gamma irradiation, respectively. In particular the internal gas pressures resulting from water hydrolysis under gamma irradiation seem to affect concrete shrinkage and creep in some measure.

2.1. Physical Interpretation of the Experimental Results. Because the shielding properties of concrete are found to be driven mainly by the selection of the aggregates in the concrete mix; the choice must be taken in reason of the different attenuation mechanisms characterizing the specific particles to be addressed: gamma-rays or neutrons [17, 3335].

Gamma-rays are attenuated by interactions at the level of the electrons of the atoms composing the shielding medium; in particular they show having a negligible effect on the solid materials composed of ionic and metallic bonds, but they can break anisotropic chemical bonds such as covalent bonds. Typically, siliceous materials are subject to gammarays degradation because the $\mathrm{Si}-\mathrm{O}$ bond is a covalent bond. At this purpose concrete to be used as a gamma-ray shielding material should be designed to maximize the density of electrons within it. Practically, high-density iron ore aggregates are chosen at this purpose. This is the case of barytes, ferrophosphorus, magnetite, and hematite.

Instead, neutrons interact with nuclei of atoms leading to change in the lattice spacing within the material during these collision events. Therefore, neutrons have a more significant effect on dense and well-crystallized materials than on randomly structured materials with high porosity. In concrete, aggregates (coarse and fine aggregates) are in a crystallized phase, while cement paste is an amorphous phase; therefore neutron radiation causes more distortion and damage to 
the internal structure of aggregates than to that of cement paste. Moreover neutron attenuation is more effective when fast neutrons are made to collide with lightweight atoms (typically hydrogen) so hydrous aggregates are to be preferred for neutron shielding; hydrous aggregates are in fact capable of increasing the fixed-water content of concrete or retaining their water of crystallization at high temperatures. This is the case of serpentine, limonite, goethite, and bauxite.

In some cases the enrichment of minerals with certain elements, such as boron, is considered to enhance the thermal neutron attenuation of concrete and cut down secondary gamma-rays production. Boron-containing aggregates include colemanite, borocalcite, and ferroboron.

The specific effect of radiation-induced damage of aggregates is of primary interest in recent research, in view of the durability assessment of old nuclear structures.

Kelly et al. [36] and Elleuch et al. [37] emphasize the predominant role of the aggregate in the development of neutron irradiation-induced swelling and the potential creation or aggravation of damage in the surrounding paste.

Vanelstraete and Laermans [38] have shown that fast neutrons cause displacement cascades in quartz resulting in disordered regions of the crystal. For sufficiently high doses, damage regions overlap, reducing long-range ordering and resulting in amorphization of the $\mathrm{SiO}_{2}$ phase. The loss of ordering is observed as a reduction of density and increase in swelling of the quartz phase. Complete amorphization is supposed to be reached at a neutron dose greater than $2 \times$ $10^{20} \mathrm{n} / \mathrm{cm}^{2}$.

The effect of nuclear irradiation, bot neutrons, and gamma-rays on quartz was studied extensively by [39]. Crystalline quartz or $\alpha$-quartz, with specific gravity of about 2.65 is found to convert to distorted amorphous quartz with specific gravity of 2.27 under a fluence of $10^{20} \mathrm{n} / \mathrm{cm}^{2}$ for fast neutrons with an energy level $>0.1 \mathrm{MeV}$ and under a dose of $10^{12} \mathrm{~Gy}$ for beta- and gamma-rays.

Maruyama et al. [8] confirm that an expansion of siliceous aggregate takes place due to neutron collisions. Atom alignments are deformed and a part of the energy imparted by neutron collision remains as a kind of strain energy causing permanent distortion. Also, denser siliceous aggregates have a larger risk of expansion due to neutron irradiation.

Because the atomic structure of some aggregates can be converted from crystalline structure to distorted amorphous structure with an increase in volume and a decrease in weight, the loss of concrete mechanical properties is conceived to be definitely correlated with radiation-induced aggregate swelling in [10].

The antagonist effect of neutrons and gamma-rays in radiation damage must be considered: the crystalline lattice structure present in the aggregates is much more affected by neutrons than the more vitreous lattice structure of the cement paste, which is much more attacked by gamma-rays.

At this purpose, many authors $[5,17,18,40,41]$ state that different types of aggregates lead to concrete with different resistance against neutron radiation. Concrete made with flint aggregate shows considerably larger volume changes than concrete made with limestone aggregates; the phenomenon can be explained, once more, by the weakness of covalent bonds in quartz aggregates with respect to the ionic bonds in calcareous aggregates.

On the other hand, the cement paste undergoes shrinkage due to (i) the radiolysis process under gamma radiation, as illustrated above, and (ii) the evaporation of pore water under radiation heat.

So the mismatch in the volumetric change of concrete components (expansion in aggregates and shrinkage of the mortar) may cause damage at the interface between the two phases.

The uneven shrinkage properties of aggregates and cement paste may lead to loss of concrete compressive strength. While the overall volume growth of the composite compromises the tensile strength, this last is shown to decay on average of $62 \%$ and $47 \%$, respectively, for flint and limestone aggregates in the range of $2 \times 10^{19}-4 \times 10^{19} \mathrm{n} / \mathrm{cm}^{2}$ [17]. According to [21] a neutron fluence of less than $10^{19} \mathrm{n} / \mathrm{cm}^{2}$ does not lead to a volume increase of the irradiated samples; rather in this range the volume change of irradiated samples is the expected shrinkage due to temperature exposure of the specimens.

The conversion of crystalline quartz into distorted quartz has a twofold detrimental consequence: (i) microcracking due to differential volume change in the composite and (ii) higher reactivity to certain aggressive chemicals, for example, calcium hydroxide responsible in concrete of alkali-silica reactions (ASR) $[42,43]$. Both of these effects are detrimental to the long-term performance of irradiated concrete.

2.2. Radiation-Induced Effects on ASR. The alkali-silica reaction evolves in concrete as follows: $\mathrm{OH}^{-}$ions present in the alkaline solution in the micropores of concrete react with $\mathrm{SiO}_{2}$ in aggregates to perform the scission of the $\mathrm{Si}-\mathrm{O}$ bonds and the subsequent expansion of the aggregates by hydration of $\mathrm{SiO}_{2}$. The consumption of $\mathrm{OH}^{-}$ions due to hydrolysis leads to the dissolution of $\mathrm{Ca}^{2+}$ ions into the solution. The $\mathrm{Ca}^{2+}$ ions then react with hydrated $\mathrm{SiO}_{2}$ gels (ASR gel) to generate calcium silicate. Rigid calcium silicate shells typically form on the surfaces of the aggregates as the reaction by-product is generated. The alkaline solution can penetrate into the aggregates through the calcium silicate shells and dissolve $\mathrm{SiO}_{2}$ groups. Since the rigid shells prevent the deformation of the aggregates, the expansion pressure generated by the penetration of the solution is accumulated in the aggregates under the confining pressure of the silicate shells, thus leading to cracks and the final expansion of the aggregates.

ASR may be accelerated both by lattice defects in $\mathrm{SiO}_{2}$ minerals from neutrons irradiation and by the preexistence of cracks in the aggregates.

The experiments by Ichikawa and Koizumi [39], Ichikawa and Kimura [44], and Ichikawa and Miura [45] prove that nuclear radiation significantly increases the reactivity of silica-rich aggregates to alkalis; the decrease of the resistance to nuclear radiation with increasing the content of $\mathrm{SiO}_{2}$ in aggregates strongly indicates that the deterioration is due to the acceleration of ASR in concrete, according to the authors.

In particular the work in [39] shows that nuclear irradiation may change the ASR expansion potential of aggregates, which is driven by the stiffness of the ASR gel and contributes 
to the free expansion capability of the aggregate in determining the extent of damage due to ASR. In fact when the ASR gel is soft and its stiffness is low, it is able to permeate into the surrounding porous cement paste; therefore the swelling pressure is not high and the cracking potential is low. In that case a large amount of ASR gel formation may not create severe damage and cracking in concrete. On the other hand, when the ASR gel is stiff, a small amount of ASR can generate significant damage [35]. The stiffness of ASR gel depends mainly on its chemical composition, such as the ratio of $\mathrm{Na}_{2} \mathrm{O}$ to $\mathrm{SiO}_{2}$. Struble and Diamond [46] conducted a study to investigate the swelling pressure of ASR in terms of ASR chemical composition.

Though not specifically addressing ASR, the work by Vodák et al. [25] indicates that irradiation generates a succession of chemical reactions, leading to a decrease in the size of pore space and hence inhibiting concrete to absorb some of the ASR gel produced prior to expansion.

Saouma and Hariri-Ardebili [47] critically reviewed existing available literature on ASR and they conclude that radiation effects on concrete degradation are minimal for the first 40-year operation of a nuclear power plant; however they agree with [6] that a structural life extension to 60-100 years may prove problematic, though the data to fully support this concern are insufficient. The work is contextualized to Seabrook site, the first reported nuclear plant in the USA known to possibly suffer from ASR, which saw in 2010 its operating license extended from 2030 to 2050. The work is aimed at developing an aging management program for old nuclear plants; it expresses the concern of overreliance on surface crack observation and structural component testing and at the same time it encourages the development of reliable Finite Element Method (FEM) based simulations to address the long-term assessment of such structures.

Graves et al. [48] offer a comprehensive evaluation of potential aging-related degradation modes for light-water reactor materials and components. Here ASR is pointed out as one of the high-ranked causes of damage that can potentially affect in the long-term the concrete containment of nuclear plants, together with creep of the posttensioned system and irradiation of concrete itself, which, as shown, may accelerate ASR.

\section{Particle Transport Simulations for Nuclear Radiation Problems}

The description of all the possible interactions between a radiation particle and the absorber medium is the topic of the so-called transport theory, a special branch of statistical mechanics.

Accurate calculations of particle transport are needed, nowadays more than in the past, for many reasons.

(i) The necessity to develop theoretical models of radiation transport also for particle energies above $20 \mathrm{MeV}$ : attenuation coefficients, such as neutron cross sections, need to be evaluated for such energies, while complete libraries are available for a representative set of nuclides up to $20 \mathrm{MeV}$ [49]. Neutrons are known to be easily transported over many energy decades, from the hundreds of $\mathrm{MeV}$ down to the meV range; therefore the knowledge of the cross section over the whole energy range is a concern.

Many new facilities, working at high energy, have been recently built or are planned, in order to perform experiments in this sense, to calibrate and validate predictive theoretical models.

(ii) The need to accurately describe the proton- and neutron-induced interaction mechanisms: a number of new applications in the last fifteen years are devoted to the production of intense neutron fluxes by protons impinging on a thick target of a heavy element (mercury, lead, uranium, etc.) for the purposes of point (i), thus producing a cascade of nuclear reactions, known as spallation reactions, which involve protons, neutrons, nuclear fragments (alphaparticles, tritium, and deuterium), and secondary gammarays.

(iii) The assistance in the design of accelerator-driven systems, which are conceived as nowadays solution to the problem of the transmutation, a possible mechanism for reducing the volume and hazard of the radioactive waste (spent fuel) in nuclear power reactors: accelerator-driven systems consist of a reactor coupled with a high-intensity proton accelerator impinging on a high-density target. The spallation reactions taking place in the target result in high neutron fluxes here and in the surrounding core, which requires accurate evaluation, for shielding design purposes and for the radiological protection assessment (e.g., thermomechanical assessment and study of the activation of components and materials).

(iv) Similarly, the assistance in the design of other nuclear facilities, such as new-generation reactors (generation IV): the concept with these next-generation reactors is to use different coolants (sodium-, gas-, or liquid-metal cooling system) and to consider recycling of the fuel, in order to both reach higher power (improved efficiency) and minimize the waste production. For these systems a radiation damage assessment of the materials subject to the intense neutron fluxes (fuel cladding, vessel, and cooling system) is mandatory for fulfilling safety requirements [50].

(v) The scientific knowledge of the biological effects of radiation, to study particle transport for biomedical purposes: Monte Carlo simulations of neutron transport in human tissues have been conducted in recent years, due to the difficulty in carrying out experiments, in order to determine the tissue-specific weighting factors at different energies, which are useful for the assessment of the effective dose of exposed subjects.

Two approaches are possible to quantify the main variable in transport theory, the radiation flux density, throughout a certain domain: the deterministic approach given by the solution of the Boltzmann transport equation and the statistical approach given by Monte Carlo simulations.

As for the first method, an exact solution is possible only in few cases [51, 52], but not for complex geometries; consequently much effort has been done in the development of approximations to the transport equation, both numerically and analytically. In this second case the diffusion theory and the two-group theory are worth mentioning. 
Numerically speaking, the solution of the Boltzmann equation requires (i) energy discretization of the neutron spectrum into discrete energy intervals based on a multigroup formalism; (ii) angular discretization of the angular direction of the radiation flux; (iii) space discretization or mesh, according to FEM formulation.

As regards the second methods, some software programs [53-57] are under continuous upgrade by their developers to solve radiation transport problems via the Monte Carlo technique.

3.1. Modeling Approaches for Irradiated Concrete. When addressing the specific issues involving irradiated concrete, especially with regard to its durability, ad hoc numerical methods must be defined to catch all the complex coupled mechanisms illustrated above, which might be used in conjunction with radiation transport calculations.

In the following some recent distinguishing models of radiation-related issues concerning concrete are reviewed.

Radiation-induced volumetric expansion is identified as a predominant source of cracking in the cement paste of pressurized water reactors in [58]. Based on an extensive literature review on postirradiation experimental data, the authors here developed a 2D micromechanical formulation accounting for the radiation-induced swelling of aggregates, which uses upscaling techniques to address the macroscopic scale starting from the material scale (aggregates and cement paste), that is, homogenization theory applied to random media. Mechanical properties of concrete vary at varying neutron fluence; no gamma-rays effects are considered nor thermohydraulic mechanisms in concrete.

In a companion study Giorla et al. [59] proposed a 2D FEM model of concrete at the mesoscale, where aggregates are supposed to be elastic and subject to thermal and radiation-induced swelling while the cement matrix is viscoelastic and it undergoes damage, drying shrinkage, and thermal expansion. Therefore this model embodies thermohydraulic aspects, though they are not coupled with the mechanical field because actually thermal expansion, radiation-induced volumetric expansion, and shrinkage result in imposed deformations. In particular here radiationinduced volumetric expansion is a predominant cause of the development and propagation of damage around the aggregate and surroundings.

In [60] a combined use of a thermohydromechanical FEM model with a Monte Carlo code is suggested to analyse radiation damage on a real nuclear facility under its design operational life. Damage is here made to depend on the neutron fluence based on Hilsdorf and coauthors' data [5], with all the limitations discussed in Section 1; the model does not account for a specific radiation-induced volumetric expansion but it allows catching the thermal aspect of the phenomenon related to the energy deposition due to radiation attenuation together with its implications on the hydraulic field.

A chemomechanical approach to model aggregate dissolution and product precipitation in a microstructural reaction-transport environment has been used in [61] to simulate near-surface degradation of Portland cement paste in contact with a sodium sulfate solution. With this tool microstructural changes due to new solids in a confinedgrowth condition are responsible for localized stress and damage in a coupled way.

So far, then, in both the theoretical and the numerical field no fully coupled radiothermohydromechanical models have been accomplished yet to simulate the deterioration mechanism of irradiated concrete, though an approach in this sense has been proposed in [7], where the mismatch in the volume changes between cement paste and aggregate is pointed out as the main cause of cracking. For the sake of completeness, the proposed model nowadays could take also advantage of the recent advances on microscopic modeling in the field of fracture mechanics and crack propagation [6266]. Recent theoretical studies and benchmark examples of engineering interest at the nanoscale can be found in [6769], also in the context of thermoelasticity and viscoelasticity [70].

3.2. Modeling Approaches for ASR. Though not always addressing radiation-induced effects specifically, a considerable interest in modeling ASR for concrete durability investigations is found in literature. The effort in the past years up to now was spent on the development of both: macroscopic models and micro-/mesoscopic models.

The first are aimed at investigating the overall structural behavior of entire concrete elements in their real context, while the latter look at the phenomenon at a material scale, often coupling the mechanical field with the chemical reactions involved in the ASR gel production.

The work by Ulm et al. [71] belongs to the first kind of approach. They developed a chemothermomechanical model in the framework of Biot's theory, where the concrete is conceived as a two-phase material including the expansive gel and the homogenized concrete skeleton. Here the volumetric expansion of the gel is evaluated as a function of the reaction kinetics, which is influenced by temperature.

Saouma and Perotti [72] proposed a chemothermomechanical model based on two main assumptions: the volumetric expansion of the gel and its redistribution in relation of weights depending on the stress tensor.

In [73] a chemoelastic damage model, validated on the basis of the accelerated multiaxial experimental tests on small specimens, is formulated to simulate the mechanical effects of ASR in large dams.

As for the applications focusing on a lower scale, various analytical models based on empirical equations were developed to explain ASR at the mesoscale of concrete: Bazant and Steffens [74] proposed that the chemical reaction kinetics is related to the diffusion process of the reactants, leading to subsequent fracture in the characteristic unit cell of the concrete, modeled with one spherical glass particle; Multon et al. [75] developed a microscopic chemomechanical model based on damage theory in order to assess the decrease of stiffness of the mortar due to cracking caused by ASR and to quantify the related expansion. The diffusion and the fixation of alkalis are here assessed with the mass balance equation and by defining a threshold alkalis concentration, above which the formation of the gel is meant to start. 
An analytical model, for which a numerical solution is provided, is studied in [76]. The formulation is based on the description of the transport and reaction of alkalis and calcium ions within a relative elementary volume, particularly taking into account the influence of the reactive aggregate size grading on ASR evolution.

Comby-Peyrot et al. [77] introduced a 3D mesoscopic FEM model of concrete as a biphasic medium with coarse aggregates randomly embedded in the cement matrix. Damage in the cement matrix is caused by the phenomenon of isotropic dilation of the reactive aggregates, specifically induced by ASR.

Dunant and Scrivener [78] developed a 2D mesoscale extended finite element (XFEM) model which defines the geometry of the gel swelling by updating the enrichment function. A specific damage parameter accounts for triggering of the fracture in the aggregates, due to growing gel pockets in them.

Alnaggar et al. [79] proposed a mesoscale formulation of ASR deterioration in concrete specimens via the Lattice Discrete Particle Model (LDPM), in a chemothermomechanical way, including nonuniform expansions, expansion transfer, and heterogeneous cracking.

In [80] 3D micro-CT scans of the microscale hardened cement paste are used to calibrate a coupled chemothermohydromechanical FEM model in which the gel is assumed to be produced in the micropores of the paste and it exerts uniform pressure on the neighborhood. The expansion coefficient of the gel at the microscale was obtained through a twostep homogenization approach, thus enabling correlating microscale damage and macroscale failure.

These references are not exhaustive; however; a comprehensive review of modeling of ASR in concrete is provided in $[81,82]$.

\section{Conclusions}

Concerns over aged nuclear power stations are mounting in several countries today. This is the primary reason for a renewed interest in radiation damage assessment of biological concrete shields in such facilities.

The behavior of irradiated concrete has reached up to date commonly accepted explanations. Particularly, cement paste and aggregates are known to behave differently under radiation exposure conditions: cement paste shrinks under drying conditions, the decrease in volume being only partially covered by the thermal expansion due to high temperatures arisen by associated radiation heat. Shrinkage of the cement paste is mostly explained by water hydrolysis and water evaporation occurring under gamma-ray irradiation. On the other hand, aggregates expand due to accumulation of defects in their crystal structures generated mainly by neutron collisions. This uneven volume expansion between cement matrix and aggregates is pointed out as the primary cause of damage in irradiated cementitious materials, to date, based on the available experimental studies. Much of the research related to this topic was conducted from the 1960s to the 1970s in support of the development of prestressed concrete reactor vessels for high temperature reactors and radioactive waste storage facilities.

The need for long-term predictions of concrete shielding of old reactors has enhanced the effort towards the modeling of the related deterioration mechanisms, both theoretically and numerically. The most relevant features which allow fully addressing the whole complex physical process are here summarized and the recent achievements in matter of irradiated concrete modeling are reviewed.

In this framework further research is recommended aimed at consolidating the new outstanding micro-/mesomechanical models in order to (i) fill the knowledge gaps from past experiments and (ii) possibly allow the integration of radiation-induced effects into the chemothermohydromechanical aspects of the problem in a coupled way.

\section{Competing Interests}

The author declares no competing interests.

\section{References}

[1] http://world-nuclear.org/info/Facts-and-Figures/World-Nuclear-Power-Reactors-and-Uranium-Requirements/.

[2] http://www.carbonbrief.org/the-trouble-with-europes-ageingnuclear-power-plants.

[3] https://euobserver.com/beyond-brussels/129795.

[4] http://uk.reuters.com/article/us-europe-nuclear-power-insightidUKKBN0GH05U20140818.

[5] H. K. Hilsdorf, J. Kropp, and H. J. Koch, "The effects of nuclear radiation on the mechanical properties of concrete," in Proceedings of the Douglas McHenry International Symposium on Concrete and Concrete Structures, ACI SP 55-10, pp. 223-251, American Concrete Institute, Mexico City, Mexico, 1978.

[6] K. Fujiwara, M. Ito, M. Sasanuma et al., "Experimental study of the effect of radiation expo-sure to concrete," in Proceedings of the 20th International Conference on Structural Mechanics in Reactor Technology (SMIRT '09), paper 1891, SMIRT20-Division I, Espoo, Finland, 2009.

[7] I. Maruyama, O. Kontani, A. Ishizawa, M. Takizawa, and O. Sato, "Development of system for evaluating concrete strength deterioration due to radiation and resultant heat," IAEA-CN194-093, Topic: 3-6 Non-metallic material Ageing Management, 2012.

[8] I. Maruyama, O. Kontani, S. Sawada, O. Sato, G. Igarashi, and M. Takizawa, "Evaluation of irradiation effects on concrete structure-background and preparation of neutron irradiation test," in Proceedings of the ASME Power Conference (POWER '13), no. 98114, 9 pages, ASME, Boston, Mass, USA, August 2013.

[9] O. Kontani, S. Sawada, I. Maruyama, M. Takizawa, and O. Sato, "Evaluation of irradiation effects on concrete structuregamma-ray irradiation tests on cement paste," in Proceedings of the ASME 2013 Power Conference (POWER '13), no. 98099, ASME, Boston, Mass, USA, August 2013.

[10] K. G. Field, I. Remec, and Y. Le Pape, "Radiation effects in concrete for nuclear power plants-part I: quantification of radiation exposure and radiation effects," Nuclear Engineering and Design, vol. 282, pp. 126-143, 2015.

[11] D. S. Billington, "Radiation damage in reactor materials," in Proceedings of the International Conference on the Peaceful Uses 
of Atomic Energy, vol. 7 of Nuclear Chemistry and Effects of Irradiation, pp. 421-432, United Nations, Geneva, Switzerland, 1955.

[12] F. E. Faris, "The effect of irradiation on structural materials," in Proceedings of the International Conference on the Peaceful Uses of Atomic Energy, vol. 7 of Nuclear Chemistry and Effects of Irradiation, pp. 484-489, United Nations, Geneva, Switzerland, August 1955.

[13] S. T. Konobeevsky, N. F. Pravdyuk, and V. I. Kutaitsev, "The effect of irradiation on the structure and properties of structural materials," in Proceedings of the International Conference on the Peaceful Uses of Atomic Energy, vol. 7 of Nuclear Chemistry and Effects of Irradiation, pp. 479-483, United Nations, Geneva, Switzerland, August 1955.

[14] A. Holmes-Siedle and L. Adams, Handbook of Radiation Effects, Oxford University Press, New York, NY, USA, 2002.

[15] B. T. Price, C. C. Horton, and K. T. Spinney, Radiation Shielding, Pergamon Press, London, UK, 1957.

[16] R. G. Jaeger, Engineering Compendium on Radiation Shielding, vol. 2, Springer, Berlin, Germany, 1970.

[17] M. F. Kaplan, Concrete Radiation Shielding: Nuclear Physics, Concrete Properties, Design, and Construction, John Wiley \& Sons, New York, NY, USA, 1989.

[18] B. S. Gray, "The effect of reactor radiation on cements and concrete," in Proceedings of the Conference on Prestressed Concrete Pressure Vessels, pp. 17-39, Commission of European Communities, Luxembourg City, Luxembourg, 1972.

[19] S. C. Alexander, Effects of Irradiation on Concrete: Final Results, Atomic Energy Research Establishment, United Kingdom Atomic Energy Authority, Harwell, UK, 1963.

[20] B. Stoces, P. Otopal, V. Juricka, and J. Gabriel, "Effect of radiation on the mechanical properties of concrete," Tech. Rep. UJV2390.R,T, Translated from the Czech, 1970.

[21] S. Granata and A. Montagnini, "Studies on behavior of concretes under irradiation," in Concrete for Nuclear Reactors, vol. 2 of Special Publication SP-34, pp. 1163-1172, American Concrete Institute, 1972.

[22] B. Nowakowski, "Influence of penetrating ionizing radiation on the curing of grouts and cement mortars," Building Science, vol. 7, no. 4, pp. 271-276, 1972.

[23] J. F. Sommers, "Gamma radiation damage of structural concrete immersed in water," Health Physics, vol. 16, no. 4, pp. 503-508, 1969.

[24] P. Soo and L. M. Milian, "The effect of gamma radiation on the strength of Portland cement mortars," Journal of Materials Science Letters, vol. 20, no. 14, pp. 1345-1348, 2001.

[25] F. Vodák, K. Trtík, V. Sopko, O. Kapičková, and P. Demo, "Effect of $\gamma$-irradiation on strength of concrete for nuclearsafety structures," Cement and Concrete Research, vol. 35, no. 7, pp. 1447-1451, 2005.

[26] F. Vodák, V. Vydra, K. Trtík, and O. Kapičková, "Effect of $\gamma$ irradiation on properties of hardened cement paste," Materials and Structures, vol. 44, no. 1, pp. 101-107, 2011.

[27] A. Lowińska-Kluge and P. Piszora, "Effect of gamma irradiation on cement composites observed with XRD and SEM methods in the range of radiation dose 0-1409 MGy," Acta Physica Polonica A, vol. 114, no. 2, pp. 399-411, 2008.

[28] P. Bouniol and A. Aspart, "Disappearance of oxygen in concrete under irradiation: the role of peroxides in radiolysis," Cement and Concrete Research, vol. 28, no. 11, pp. 1669-1681, 1998.
[29] P. Bouniol and E. Bjergbakke, "A comprehensive model to describe radiolytic processes in cement medium," Journal of Nuclear Materials, vol. 372, no. 1, pp. 1-15, 2008.

[30] P. Bouniol, B. Muzeau, and V. Dauvois, "Experimental evidence of the influence of iron on pore water radiolysis in cement-based materials," Journal of Nuclear Materials, vol. 437, no. 1-3, pp. 208-215, 2013.

[31] D. J. Naus, "A compilation of elevated temperature concrete material property data and information for use in assessments of nuclear power plant reinforced concrete structures," Tech. Rep. NUREG/CR-7031 ORNL/TM-2009/175, US Nuclear Regulatory Commission, Oak Ridge National Laboratory, 2010.

[32] D. McDowall, "The effects of gamma radiation on the creep properties of concrete," in Proceedings of the Information Exchange Meeting on "Results of Concrete Irradiation Programs", EUR 4751 f-e, pp. 55-69, Commission des Communautés Européennes, Brussels, Belgium, 1971.

[33] T. Rockwell, Reactor Shielding Design Manual, McGraw-Hill, New York, NY, USA, 1956.

[34] A. N. Komarovskii, Shielding Materials for Nuclear Reactors, Pergamon Press, London, UK, 1961.

[35] K. William, Y. Xi, and D. Naus, "A review of the effects of radiation on microstructure and properties of concretes used in nuclear power plants," Tech. Rep. NUREG/CR-7171 ORNL/TM2013/263, US Nuclear Regulatory Commission, Oak Ridge National Laboratory, 2013.

[36] B. Kelly, J. Brocklehurst, D. Mottershead, and S. McNearney, "The effects of reactor radiation on concrete," in Proceedings of the 2nd Information Meeting on Prestressed Concrete and Reactor Pressure Vessels and Their Thermal Isolation, EUR-4531, pp. 237265, Commission des Communautés Européennes, Brussels, Belgium, 1969.

[37] M. F. Elleuch, F. Dubois, and J. Rappeneau, "Effects of neutron radiation on special concretes and their components," in ACI Special Publication SP-34: Concrete for Nuclear Reactors, Paper SP34-51, pp. 1071-1108, 1972.

[38] A. Vanelstraete and C. Laermans, “Tunneling states in neutronirradiated quartz: measurements of the ultrasonic attenuation and velocity change," Physical Review B, vol. 42, no. 9, pp. 5842$5854,1990$.

[39] T. Ichikawa and H. Koizumi, "Possibility of radiation-induced degradation of concrete by alkali-silica reaction of aggregates," Journal of Nuclear Science and Technology, vol. 39, no. 8, pp. 880884, 2002.

[40] D. Campbell-Allen, E. W. E. Low, and H. Roper, "An investigation on the effect of elevated temperatures on concrete for reactor vessels," Nuclear Structural Engineering, vol. 2, no. 4, pp. 382-388, 1965.

[41] D. J. Naus, "Primer on durability of nuclear power plant reinforced concrete structures: a review of pertinent factors," Tech. Rep. NUREG/CR-6927 ORNL/TM-2006/529, US Nuclear Regulatory Commission, Oak Ridge National Laboratory, 2007.

[42] A. F. Cohen, "Low-temperature thermal conductivity in neutron irradiated vitreous silica," Journal of Applied Physics, vol. 29, no. 3, pp. 591-593, 1958.

[43] L. W. Hobbs and M. R. Pascucci, "Radiolysis and defect structure in electron-irradiated $\alpha$-quartz," Journal de Physique Colloques, vol. 41, no. 7, pp. 237-242, 1979.

[44] T. Ichikawa and T. Kimura, "Effect of nuclear radiation on alkali-silica reaction of concrete," Journal of Nuclear Science and Technology, vol. 44, no. 10, pp. 1281-1284, 2007. 
[45] T. Ichikawa and M. Miura, "Modified model of alkali-silica reaction," Cement and Concrete Research, vol. 37, no. 9, pp. 12911297, 2007.

[46] L. Struble and S. Diamond, "Unstable swelling behaviour of alkali silica gels," Cement and Concrete Research, vol. 11, no. 4, pp. 611-617, 1981.

[47] V. E. Saouma and M. A. Hariri-Ardebili, "A proposed aging management program for alkali silica reactions in a nuclear power plant," Nuclear Engineering and Design, vol. 277, pp. 248264, 2014.

[48] H. Graves, Y. Le Pape, D. Naus et al., "Expanded materials degradation assessment (EMDA), 4: aging of concrete," Tech. Rep. NUREG/CR-7153, ORNL/TM-2011/545, US Nuclear Regulatory Commission, Oak Ridge National Laboratory, 2013.

[49] P. Vaz, "Neutron transport simulation (selected topics)," Radiation Physics and Chemistry, vol. 78, no. 10, pp. 829-842, 2009.

[50] A. Alemberti, "Verso i reattori di IV generazione," in Proceedings of the Workshop: IV Generation Nuclear Reactors: Which Issues for Metals? AIM (Italian Association of Metallurgy) with Ansaldo Nucleare and IENI (National Council for Research, Institute for Energy and Interphases), Genova, Italy, February 2011, (Italian).

[51] J. R. Harrison, Nuclear Reactor Shielding, Temple Press, London, UK, 1958.

[52] J. K. Shultis and R. E. Faw, Radiation Shielding, Prentice Hall, New York, NY, USA, 1996.

[53] D. B. Pelowitz, Ed., "MCNPX user's manual, version 2.7.0”, Tech. Rep. LA-CP-11-0438, Los Alamos National Laboratory, 2011.

[54] D. B. Pelowitz, J. W. Durkee, J. S. Elson et al., "MCNPX 2.7.0 extensions," Tech. Rep. LA-UR-11-02295, Los Alamos National Laboratory, 2011.

[55] S. Agostinelli, "Geant4-a simulation toolkit," Nuclear Instruments and Methods in Physics Research Section A: Accelerators, Spectrometers, Detectors and Associated Equipment, vol. 506, no. 3, pp. 250-303, 2003.

[56] J. Allison, K. Amako, J. Apostolakis et al., "Geant4 developments and applications," IEEE Transactions on Nuclear Science, vol. 53, no. 1, pp. 270-278, 2006.

[57] A. Ferrari, P. R. Sala, A. Fass, J. Ranft, and A. Fassò, "FLUKA: a multi-particle transport code," Tech. Rep. CERN-2005-10, INFN/TC_05/11, SLAC-R-773, 2011.

[58] Y. Le Pape, K. G. Field, and I. Remec, "Radiation effects in concrete for nuclear power plants, part II: perspective from micromechanical modeling," Nuclear Engineering and Design, vol. 282, pp. 144-157, 2015.

[59] A. Giorla, M. Vaitová, Y. Le Pape, and P. Štemberk, "Mesoscale modeling of irradiated concrete in test reactor," Nuclear Engineering and Design, vol. 295, pp. 59-73, 2015.

[60] B. Pomaro, V. A. Salomoni, F. Gramegna, G. Prete, and C. E. Majorana, "Radiation damage evaluation on concrete shielding for nuclear physics experiments," Annals of Solid and Structural Mechanics, vol. 2, no. 2-4, pp. 123-142, 2011.

[61] P. Feng, E. J. Garboczi, C. Miao, and J. W. Bullard, "Microstructural origins of cement paste degradation by external sulfate attack," Construction and Building Materials, vol. 96, pp. 391403, 2015.

[62] A. M. Tarantino, "The singular equilibrium field at the notchtip of a compressible material in finite elastostatics," Journal of Applied Mathematics and Physics, vol. 48, no. 3, pp. 370-388, 1997.
[63] A. M. Tarantino, "On extreme thinning at the notch tip of a neoHookean sheet," The Quarterly Journal of Mechanics and Applied Mathematics, vol. 51, no. 2, pp. 179-190, 1998.

[64] A. M. Tarantino, "On the finite motions generated by a mode I propagating crack," Journal of Elasticity, vol. 57, no. 2, pp. 85103, 1999.

[65] A. M. Tarantino, "Crack propagation in finite elastodynamics," Mathematics and Mechanics of Solids, vol. 10, no. 6, pp. 577-601, 2005.

[66] A. Caporale, L. Feo, and R. Luciano, "Damage mechanics of cement concrete modeled as a four-phase composite," Composites Part B: Engineering, vol. 65, pp. 124-130, 2014.

[67] R. Barretta, L. Feo, R. Luciano, and F. Marotti de Sciarra, "An Eringen-like model for Timoshenko nanobeams," Composite Structures, vol. 139, pp. 104-110, 2016.

[68] R. Barretta, L. Feo, R. Luciano, and F. Marotti de Sciarra, "A gradient Eringen model for functionally graded nanorods," Composite Structures, vol. 131, pp. 1124-1131, 2015.

[69] R. Barretta, L. Feo, R. Luciano, and F. Marotti de Sciarra, "Variational formulations for functionally graded nonlocal Bernoulli-Euler nanobeams," Composite Structures, vol. 129, pp. 80-89, 2015.

[70] R. Barretta, L. Feo, and R. Luciano, "Torsion of functionally graded nonlocal viscoelastic circular nanobeams," Composites Part B: Engineering, vol. 72, pp. 217-222, 2015.

[71] F.-J. Ulm, O. Coussy, L. Kefei, and C. Larive, "Thermo-chemomechanics of ASR expansion in concrete structures," Journal of Engineering Mechanics, vol. 126, no. 3, pp. 233-242, 2000.

[72] V. Saouma and L. Perotti, "Constitutive model for alkaliaggregate reactions," ACI Materials Journal, vol. 103, no. 3, pp. 194-202, 2006.

[73] C. Comi, R. Fedele, and U. Perego, "A chemo-thermo-damage model for the analysis of concrete dams affected by alkali-silica reaction," Mechanics of Materials, vol. 41, no. 3, pp. 210-230, 2009.

[74] Z. P. Bazant and A. Steffens, "Mathematical model for kinetics of alkali-silica reaction in concrete," Cement and Concrete Research, vol. 30, no. 3, pp. 419-428, 2000.

[75] S. Multon, A. Sellier, and M. Cyr, "Chemo-mechanical modeling for prediction of alkali silica reaction (ASR) expansion," Cement and Concrete Research, vol. 39, no. 6, pp. 490-500, 2009.

[76] S. Poyet, A. Sellier, B. Capra et al., "Chemical modelling of alkali silica reaction: influence of the reactive aggregate size distribution," Materials and Structures, vol. 40, no. 2, pp. 229239, 2007.

[77] I. Comby-Peyrot, F. Bernard, P.-O. Bouchard, F. Bay, and E. Garcia-Diaz, "Development and validation of a 3D computational tool to describe concrete behaviour at mesoscale. Application to the alkali-silica reaction," Computational Materials Science, vol. 46, no. 4, pp. 1163-1177, 2009.

[78] C. F. Dunant and K. L. Scrivener, "Micro-mechanical modelling of alkali-silica-reaction-induced degradation using the AMIE framework," Cement and Concrete Research, vol. 40, no. 4, pp. 517-525, 2010.

[79] M. Alnaggar, G. Cusatis, and G. Di Luzio, "A discrete model for alkali-silica-reaction in concrete," in Proceedings of the 8th International Conference on Fracture Mechanics of Concrete and Concrete Structures (FraMCoS '12), J. G. M. Van Mier, G. Ruiz, C. Andrade, R. C. Yu, and X. X. Zhang, Eds., 2012. 
[80] T. Wu, I. Temizer, and P. Wriggers, "Multiscale hydro-thermochemo-mechanical coupling: Application to alkali-silica reaction," Computational Materials Science, vol. 84, pp. 381-395, 2014.

[81] J. W. Pan, Y. T. Feng, J. T. Wang, Q. C. Sun, C. H. Zhang, and D. R. J. Owen, "Modeling of alkali-silica reaction in concrete: a review," Frontiers of Structural and Civil Engineering, vol. 6, no. 1, pp. 1-18, 2012.

[82] L. F. M. Sanchez, S. Multon, A. Sellier, M. Cyr, B. Fournier, and M. Jolin, "Comparative study of a chemo-mechanical modeling for alkali silica reaction (ASR) with experimental evidences," Construction and Building Materials, vol. 72, pp. 301-315, 2014. 


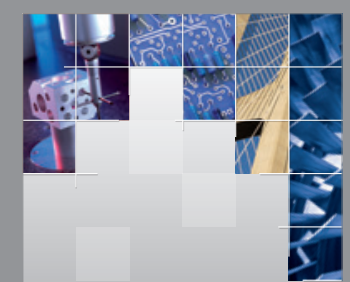

\section{Enfincering}
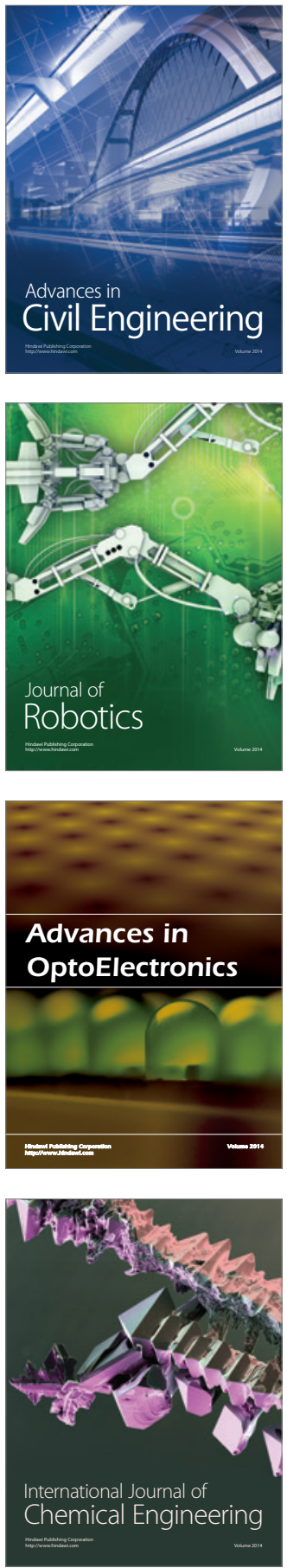

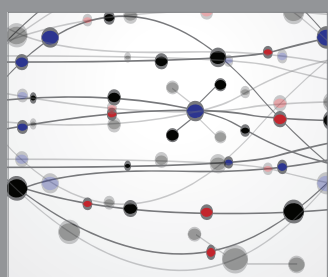

The Scientific World Journal

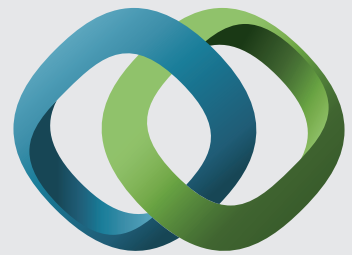

\section{Hindawi}

Submit your manuscripts at

http://www.hindawi.com
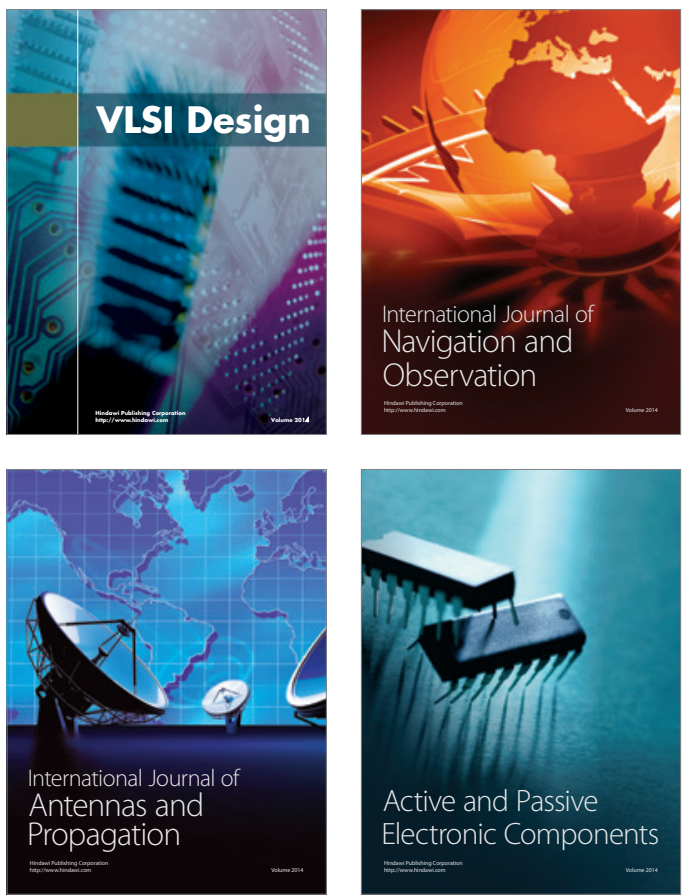
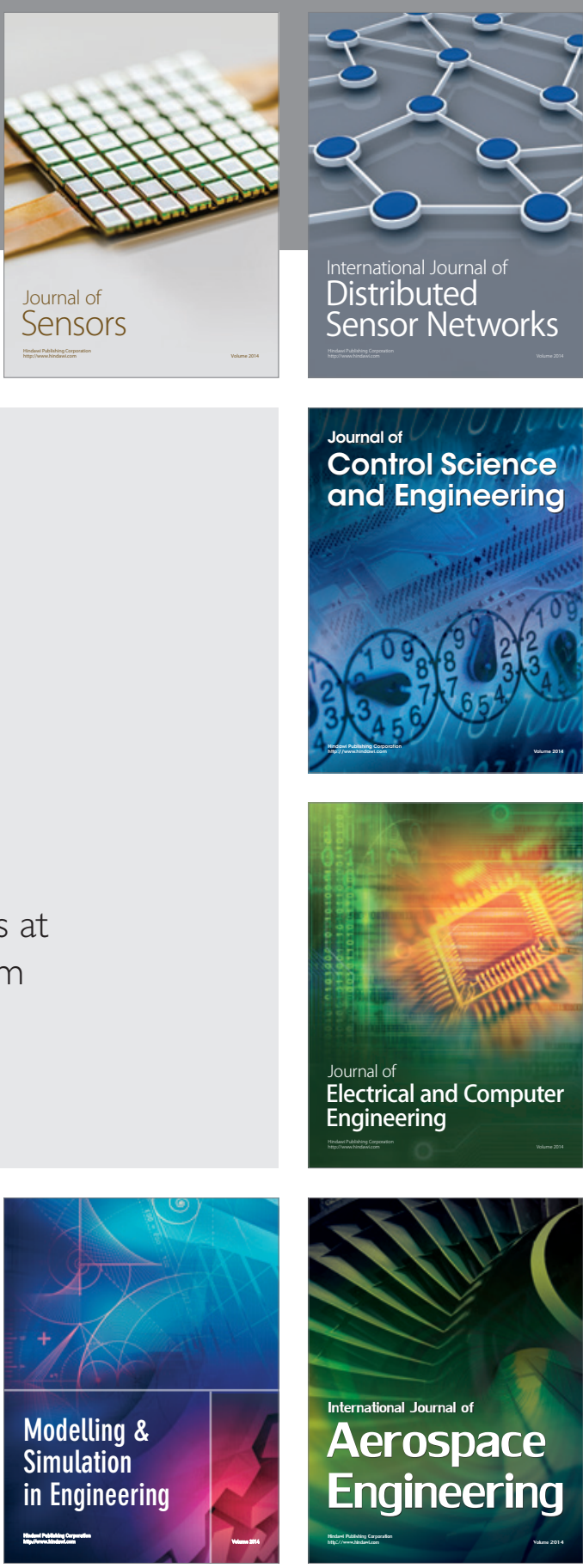

International Journal of

Distributed

Sensor Networks

Journal of

Control Science

and Engineering
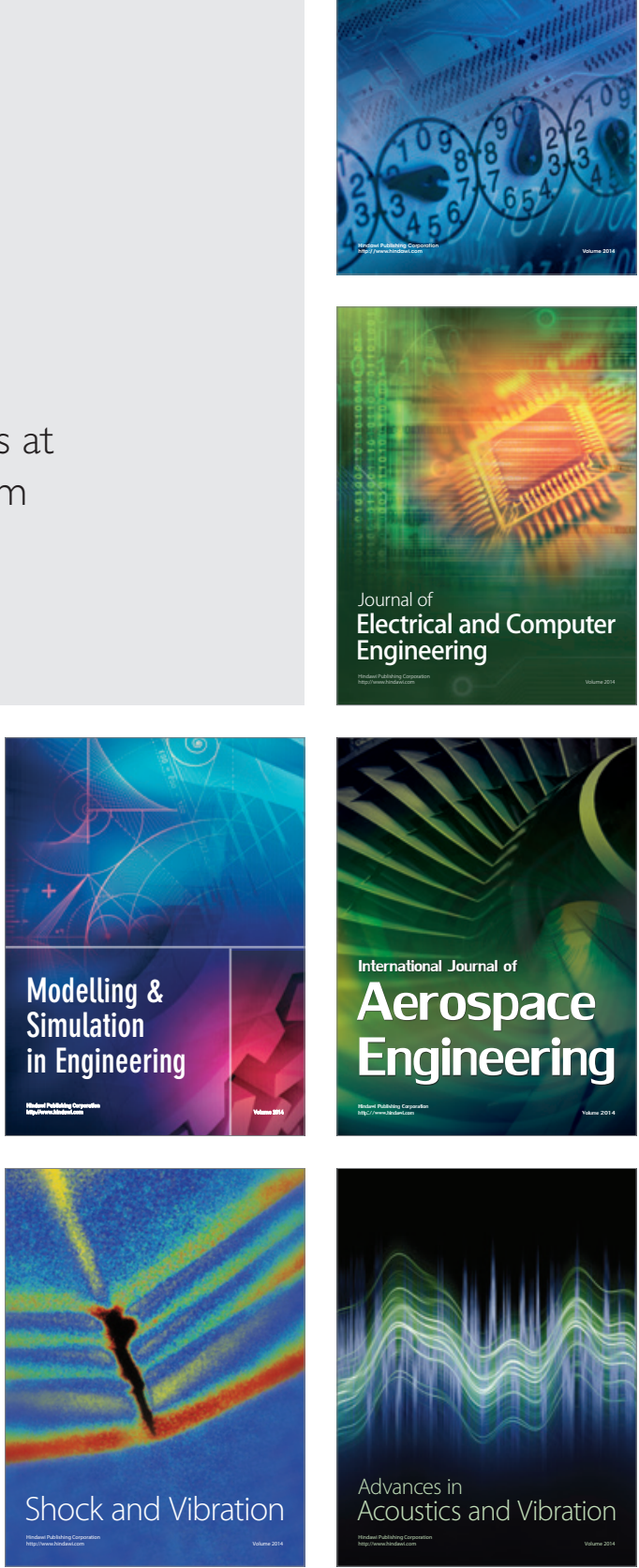